\title{
Extended halos and intracluster light using Planetary Nebulae as tracers in nearby clusters
}

\author{
Magda Arnaboldi ${ }^{1,2}$ \\ ${ }^{1}$ European Southern Observatory, K. Schwarszchild-str. 2, 85748 Garching, Germany \\ email: marnabol@eso.org \\ ${ }^{2}$ INAF, Oss. Astr. di Pino Torinese, 10025 Pino Torinese, Italy
}

\begin{abstract}
Since the first detection of intracluster planetary nebulae in 1996, imaging and spectroscopic surveys identified such stars to trace the radial extent and the kinematics of diffuse light in clusters. This topic of research is tightly linked with the studies of galaxy formation and evolution in dense environment, as the spatial distribution and kinematics of planetary nebulae in the outermost regions of galaxies and in the cluster cores is relevant for setting constraints on cosmological simulations. In this sense, extragalactic planetary nebulae play a very important role in the near-field cosmology, in order to measure the integrated mass as function of radius and the orbital distribution of stars in structures placed in the densest regions of the nearby universe.
\end{abstract}

Keywords. planetary nebulae: general. galaxies: halos, clusters: general, individual (Virgo, Hydra, Coma); elliptical and lenticular, $\mathrm{cD}$; kinematics and dynamics; galaxies individual: (NGC 4486, NGC 3311, NGC 4784, NGC 4889)

\section{Introduction}

Hierarchical structure formation theories predict that massive galaxy clusters are built through the infall of matter, i.e. galaxies, groups and sub-clusters, along large scale filaments (White \& Rees (1978)). Since mass accretion is still active today, nearby galaxy clusters may be at different epochs of their mass assembly, and they may still be in an unmixed state.

The gravitational forces acting on galaxies during cluster formation and its evolution unbind a fraction of their stars, which then end-up orbiting in the intracluster region. This cluster-driven diffuse stellar component is often referred to as "intracluster light (ICL)". Numerical simulations show that the amount of ICL in clusters depends on the cluster mass and dynamical state (Cooper et al. 2015). A more massive, older, dynamically evolved cluster may contain a larger amount of ICL than a less massive or a dynamically younger system (Rudick et al. 2006; Murante et al. 2007). Furthermore, in a highly evolved dynamically old cluster, the ICL morphology would be more diffuse, with relative few streams, while a dynamically young cluster dominated by groups still in the process of merging is likely to be dominated by ICL found in streams (Rudick et al. 2009). Therefore the study of the amount, distribution and kinematics of the ICL may provide information on the cluster accretion history and evolutionary state, as well as about the evolution of cluster galaxies.

Several studies have mapped the distribution of the ICL in nearby clusters (see Mihos 2015 for a review): since the serendipitous discovery of three intracluster planetary nebulae $(\mathrm{ICPNs})$ moving at $v_{\text {mean }}=1400 \mathrm{~km} \mathrm{~s}^{-1}$ along the line-of-sight of NGC 4406 $\left(v_{\text {sys }}=-240 \mathrm{~km} \mathrm{~s}^{-1}\right)$ in the Virgo cluster core (Arnaboldi et al. 1996), these stellar 
tracers have been used to map the spatial distribution and kinematics of the diffuse light in clusters. In what follows, the physical properties of the ICPNs samples in the nearby clusters, i.e. Virgo, Hydra I and Coma, will be outlined and compared.

\section{The Virgo cluster}

Several studies investigated the properties of the ICL in the core of the nearby Virgo cluster (Feldmeier et al. 1998, 2003, 2004; Arnaboldi et al. 2002, 2003; Aguerri et al. 2005) and around M49 (Feldmeier et al. 2004). Expanding on these earlier survey works, Castro-Rodriguez et al. (2009) completed a survey campaign of the ICL distribution on larger scales, outside the center of the Virgo cluster. In total, they covered more than 3 square degrees in Virgo, at eleven different positions in the cluster and at distances between 80 arcmin and some 100 arcmin from the Virgo cluster center. In several of these fields, the ICL is at least two magnitudes fainter than in the Virgo core region.

These new results are in agreement with observations of intermediate redshift clusters (Zibetti et al. 2005; D'Souza et al. 2014), and with the results of high resolution hydrodynamical simulations of cluster formation in a $\lambda$ CDM universe (see also Murante et al. 2004), which predict that the ICL is more centrally concentrated than cluster galaxies and that the largest portion of the ICL is formed during the assembly of the most luminous cluster galaxies.

\section{Kinematics of planetary nebulae in the Virgo cluster core}

Early attempts to measure spectra for ICPN in Virgo (Arnaboldi et al. 2003, 2004; Doherty et al. 2009) provided spectra for 52 single PNs around M87 and the Virgo core. These single spectra showed the [OIII $] \lambda 4959 / 5007 \AA$ double emissions, confirming the identification of these emission line candidates and their association with the Virgo cluster. Furthermore the measured expansion velocities of the [OIII] envelope for these bright PNs is similar to those measured for the bright PNs in the Milky Way bulge (Arnaboldi et al. 2008).

The sparsely sampled projected phase-space of these PNs that was built from their lineof-sight velocity $\left(v_{L O S}\right)$ vs. radial distance from the center of M87 was probing regions with different kinematics. The halo of M87 was clearly detected as a high density region centered around its systemic velocity $\left(1300 \mathrm{~km} \mathrm{~s}^{-1}\right)$, and a broad line-of-sight velocity distribution (LOSVD) was measured at distances of 2000 arcsec and larger. Such broad LOSVD is reminiscent of the velocity distribution of the galaxies in the Virgo cluster (Binggeli et al. 1987).

The recent, extended PN survey carried out by Longobardi et al. (2013) represents a major increase with respect with previous sample in the same M87 area of a factor 15 . With the narrow band imaging carried out with Suprime-Cam at Subaru, they detected $688 \mathrm{PN}$ candidates in an area of nearly $0.5 \mathrm{sq}$-deg covering M87. The spectroscopic followup was performed with Flames at UT2 on the VLT; 287 spectra were obtained for single PNs (Longobardi et al. 2015). This sample was analyzed co-jointly with 12 PNs from Doherty et al. (2009) in the same area, giving a merged total sample of 301 PNs.

Longobardi et al. (2015) built the LOSVD for the entire sample of the spectroscopically confirmed PNs. The LOSVD displays broad asymmetric wings that cannot be matched by a single Gaussian, see Figure 5 in Longobardi et al. (2015). The entire LOSVD was fitted by a narrow Gaussian centered at the systemic velocity of M87 and $\sigma_{n} \simeq 300 \mathrm{~km}$ $\mathrm{s}^{-1}$ and a broad Gaussian component with average velocity at $v_{b} \simeq 1000 \mathrm{~km} \mathrm{~s}^{-1}$ and $\sigma_{b} \simeq 900 \mathrm{~km} \mathrm{~s}^{-1}$. This kinematical decomposition is carried out in the projected space 
space, $v_{L O S}$ vs. $R_{M a j o r}$, and PNs can be assigned either to the narrow Gaussian, i.e. the M87 halo component, or to the broad component, i.e. the ICL, via a robust sigma-clipping procedure (Longobardi et al. 2015).

Such kinematically identified PN populations showed different physical properties, in addition. Firstly, the M87 halo PNs number density profile follows the Sersic fit to the V-band surface brightness profile from Kormendy et al. (2009), while the ICPNs number density profile is consistent with a power law, $N_{I C P N}(R) \propto R^{\gamma}$ with $\gamma=[-0.34,-0.04]$. Secondly, the two PN populations have different specific frequencies with the ICL having three times larger PN yield per bolometric luminosity than the M87 halo light. Thirdly, the morphology of the PN luminosity function (PNLF) for these two components differs. The PNLF for the M87 halo sample has a steeper gradient than the M31 PNLF or the standard Ciardullo's analytical formula (Ciardullo et al. 1989), while the PNLF for the ICPN shows a "dip" which is reminiscent of a similar morphological feature detected in the SMC/LMC PN samples (Jacoby \& De Marco 2002, Reid \& Parker 2010). The similarity of the IC PNLF with those observed for low-luminosity, low metallicity, star forming irregulars support the identification of these galaxies as likely progenitors of the stars that are now found associated with the ICL in the Virgo cluster core (Longobardi et al. 2015).

\section{Detecting Planetary Nebulae beyond 20 Mpc distance}

The brightest PNs at $50 \mathrm{Mpc}$ distance have fluxes of $7.8 \times 10^{-18} \mathrm{erg} \mathrm{s}^{-1} \mathrm{~cm}^{-2}(\sim 7$ photons/min on $8 \mathrm{~m}$ tel.). In the Coma cluster, the fluxes of the brightest PNs are four times fainter. Hence the PN [OIII] $\lambda 5007 \AA$ emission cannot be detected using a narrow band filter because the sky noise in a $30-40 \AA$ window centered on the red-shifted [OIII] PN emission is of the same order as the signal from such line. A step forward for the detection of PN in elliptical galaxies in clusters at distances larger than $20 \mathrm{Mpc}$ is to decrease significantly the sky noise. This is achieved with the Multi Slit Imaging Spectroscopy Technique (MSIS; Gerhard et al. 2005).

MSIS is a blind search technique, that combines a mask of parallel multiple slits with a narrow band filter, centered on the red-shifted [OIII] $\lambda 5007 \AA$ line at the Hydra I/Coma mean systemic velocities, to obtain spectra of all PNs that lie behind the slits (Gerhard et al. 2005). The sky noise at the PN [OIII] emission line now comes from a spectral range of only a few $\AA$, depending on slit width and spectral resolution (Arnaboldi et al. 2007). Several tens of PNs were detected using the MSIS observations in the Hydra I (Ventimiglia et al. 2011) and Coma (Gerhard et al. 2007, Arnaboldi et al. 2007) clusters.

The Hydra I cluster and NGC 3311 - Ventimiglia et al. (2011) performed the MSIS observations with FORS2 at the ESO VLT of the core region of the Hydra I cluster, centered on its cD galaxy, NGC 3311. They detected a total of $56 \mathrm{PNs}$ in a single field of $100 \times 100 \mathrm{kpc}^{2}$, and analyzed the LOSVD. The PNs LOSVD in this region has several peaks, see Figure 1: in addition to a broad symmetric component centered at the systemic velocity of the cluster $\left(v_{H y d r a}=3683 \mathrm{~km} \mathrm{~s}^{-1}\right)$, two narrow peaks are detected at 1800 $\mathrm{km} \mathrm{s}^{-1}$ and $5000 \mathrm{~km} \mathrm{~s}^{-1}$. These secondary peaks unmask the presence of un-mixed stellar populations in the Hydra I core.

The spatial distribution of the PNs associated with the narrow velocity sub-component at $5000 \mathrm{~km} \mathrm{~s}^{-1}$ is superposed and concentrated on an excess of light in the North-East quadrant of NGC 3311, as detected from the two-dimensional light decomposition of the NGC 3311/NGC 3309 B band photometry, see Arnaboldi et al. (2012). On the same sky location, we detect a group of dwarf galaxies with $v_{L O S} \approx 5000 \mathrm{~km} \mathrm{~s}^{-1}$. Deep long-slit spectra obtained at the position of the dwarf galaxy HCC 026 situated at the center of 

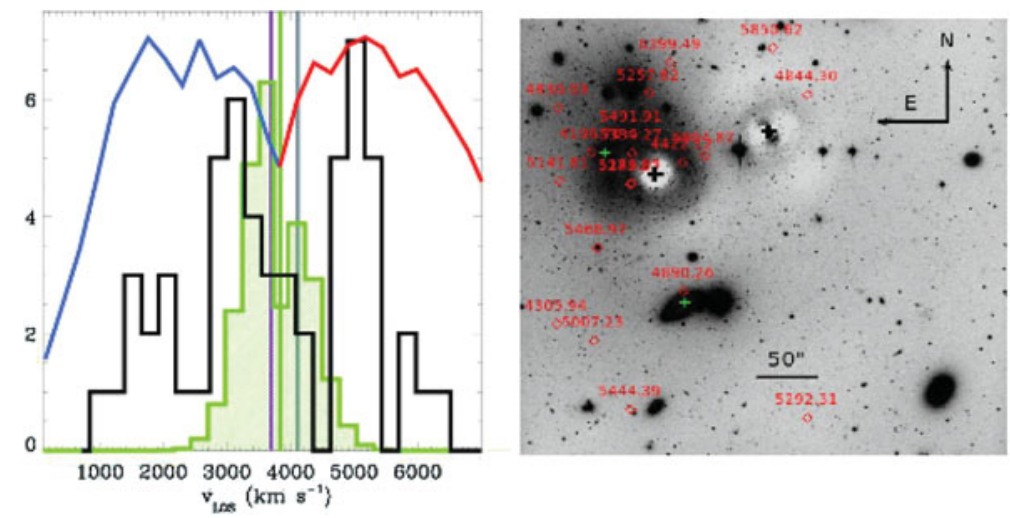

Figure 1. Left panel: the PNs LOSVD in the core of the Hydra I cluster obtained from the MSIS observations by Ventimiglia et al. (2011). The LOSVD is shown by the black line, while the blue and red curves indicate the measured transmission curves of the narrow band filters used to cover the Hydra velocities; the normalization is arbitrary. The magenta, green and gray vertical lines indicate the systemic velocity of the Hydra-I cluster, NGC 3311 and NGC 3309, respectively. The green histogram shows the simulated MSIS LOSVD for a Gaussian velocity distribution with $\sigma_{\text {core }}=464 \mathrm{~km} \mathrm{~s}^{-1}$; from Ventimiglia et al. (2011). Right panel: Positions and velocities of PNs (red diamonds); labels indicate the PNs $V_{L O S}$ in $\mathrm{km} \mathrm{s}^{-1}$ associated with the red peak at $5000 \mathrm{~km} \mathrm{~s}^{-1}$ in the PN LOSVD of the Hydra I cluster. The red peak PNs are superposed on the residual V-band image, which shows the substructures in the diffuse light in the Hydra I cluster core. The black crosses indicate the position of NGC 3311 (center) and NGC 3309 (upper right), respectively. The green crosses indicate HCC 026 and HCC 007. The FoV is $6.8^{\prime} \times 6.4^{\prime}$. From Arnaboldi et al. $(2012)$.

the light excess show absorption line features from both HCC 026 and the light excess which are consistent with $v_{L O S} \sim 5000 \mathrm{~km} \mathrm{~s}^{-1}$. Arnaboldi et al. 2012 concluded that the PNs in the $5000 \mathrm{~km} \mathrm{~s}^{-1}$ sub-component, the dwarfs galaxies and the light excess in the North-East quadrant of NGC 3311 occupy the same region of the phase-space, and are physically associated.

Hence also in the case of the relaxed Hydra-I cluster, PN kinematics, photometry and deep absorption spectra support the evidence for an accretion event whose stars are being added to both the cluster core and the halo of NGC 3311.

\section{The on-going sub-cluster merger in the Coma cluster core}

The Coma cluster is the richest and most compact of the nearby clusters, yet there is growing evidence that its formation is still on-going. A sensitive probe of this evolution is the dynamics of intracluster stars, which are unbound from galaxies while the cluster forms, according to cosmological simulations. With the MSIS technique, Gerhard et al. (2005) detected and measured the $v_{L O S}$ of 37 ICPNs associated with the diffuse stellar population of stars in the Coma cluster core, at $100 \mathrm{Mpc}$ distance. These are the most distant single stars whose spectra were acquired, in addition to cosmological supernovae stars. Gerhard et al. (2007) detected clear velocity sub-structures within a 6 arcmin diameter field centered at $\alpha(J 2000)=12: 59: 41.8 ; \delta(J 2000) 27: 53: 25.4$, nearly coincident with the field observed by Bernstein et al. (1995) and $\sim 5$ arcmin away from the $\mathrm{cD}$ galaxy NGC 4874. A sub-structure is present at $\sim 5000 \mathrm{kms}^{-1}$, probably from in-fall of a galaxy group, while the main intracluster stellar component is centered around $\sim 6500 \mathrm{kms}^{-1}, \sim 700 \mathrm{kms}^{-1}$ offset from the nearby cD galaxy NGC $4874\left(v_{\text {sys }}=7224\right.$ $\mathrm{km} \mathrm{s}^{-1}$; from NED). The kinematics and the elongated morphology of the intracluster 


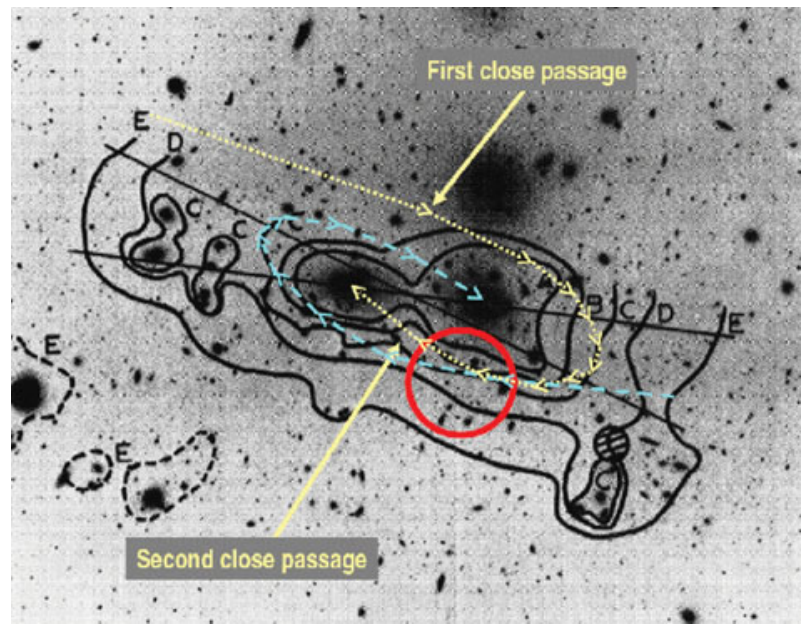

Figure 2. The position of the MSIS field (red circle) studied in Gerhard et al. (2007) on the diffuse light isodensity contours drawn by Thuan \& Kormendy 1977 in the Coma cluster core. The MSIS field is about $5^{\prime}$ south of NGC 4874. The second Coma cD galaxy NGC 4889 is $7^{\prime}$ east (to the left) of NGC 4874. The bright object north of NGC 4874 is the star which prevented Thuan \& Kormendy 1977 from reliably determining the northern parts of the isodensity curves in their photographic photometry. Note the strong elongation of the distribution of ICL in the Coma cluster core. The likely orbits of NGC 4889 and NGC 4874 up to their present positions are sketched by the yellow dotted and magenta dashed lines, respectively; see Gerhard et al. (2007).

stars (Thuan \& Kormendy 1977) show that the cluster core is in a highly dynamically evolving state. In combination with galaxy redshift and X-ray data, this argues strongly that the cluster is currently in the midst of a sub-cluster merger. The NGC 4889 subcluster is likely to have fallen into Coma from the eastern A2199 filament, in a direction nearly in the plane of the sky, meeting the NGC 4874 sub-cluster arriving from the west. The two inner sub-cluster cores are presently beyond their first and second close passage, during which the elongated distribution of diffuse light has been created, see Figure 2 . Gerhard et al. (2007) also predicted the kinematic signature expected in this scenario, and argued that the extended western X-ray arc recently discovered traces the arc shock generated by the collision between the two sub-cluster gaseous halos.

\section{Summary and Conclusions}

The kinematics of the diffuse light mapped via by extragalactic PNs provide unique information to asses the dynamical status of the nearby cluster cores. In all cases the kinematical data indicate that the galaxy halos and ICL are discrete components and the former do not blend continuously in the latter. The evidence for merging (in Coma) and accretion on the extended halos (M87, NGC 3311) indicates that the build up of the diffuse light in the cluster cores is an on-going process.

The predicted spatially averaged radial distribution of ICL from recent high resolution hydrodynamical simulations of cluster formation in $\Lambda$ CDM universe is in broad agreement with the observed radial profiles for the ICL in clusters. Furthermore predictions from these simulations indicate that the more massive progenitors dominate the diffuse light in simulated cluster close to the center (Murante et al. 2007, Puchwein et al. 2010). The prediction that the largest portion of the ICL is formed during the assembly of the 
most luminous cluster galaxies is supported by the observed ongoing mergers in Virgo, Hydra I and Coma cores.

Quantitative analysis of the ICL kinematic from cosmological simulations is on-going. Studies of the galaxy halo and ICL particles in cosmological simulations (Dolag et al. 2010, Cui et al. 2014) further support the physical distinction between the central bright galaxy and the ICL component in clusters.

\section{Acknowledgment}

The author would like to thank the organizers for the invitation to give a review on extragalactic PNs as tracers of diffuse light at the IAU FM 4.

\section{References}

Aguerri, J. A. L., Gerhard, O. E., Arnaboldi, M., Napolitano, N. R., et al. 2005, AJ, 129, 2585 Arnaboldi, M., Ventimiglia, G., Iodice, E., Gerhard, O. E. et al. 2012, A\& A, 545, 37

Arnaboldi, M., Doherty, M., Gerhard, O. E., Ciardullo, R. et al. 2008, ApJ, 674, L17

Arnaboldi, M., Gerhard, O. E., Okamura, S., Kashikawa, N. et al. 2007, PASJ, 59, 419

Arnaboldi, M., Gerhard, O. E., Aguerri, J. Alfonso, L., Freeman, K. C. et al. 2004, ApJ, 614, 33

Arnaboldi, M., Freeman, K. C., Okamura, S., Yasuda, N. et al. 2003, AJ, 125, 514

Arnaboldi, M., Aguerri, J. A. L., Napolitano, N. R., Gerhard, O. E. et al. 2002, AJ, 123, 760

Arnaboldi, M., Freeman, K. C., Mendez, R. H., Capaccioli, M. et al. 1996, ApJ, 472, 145

Bernstein, G. M., Nichol, R. C., Tyson, J. A., Ulmer, M. P., \& Wittman, D. 1995, AJ, 110, 1507

Binggeli, B., Tammann, G. A., \& Sandage, A. 1987, AJ, 94, 251

Castro-Rodriguez, N., Arnaboldi, M., Aguerri, J. A. L., Gerhard, O. et al. 2009, A\&A, 507, 621

Ciardullo, R., Jacoby, G. H., Ford, H. C., \& Neill, J. D. 1989, ApJ, 339, 53

Cooper, A. P., Gao, L., Guo, Q., Frenk, C. S., et al. 2015, MNRAS, 451, 2703

Cui, W., Murante, G., Monaco, P., Borgani, S. et al. 2014, MNRAS, 437, 816

Doherty, M., Arnaboldi, M., Das, P., Gerhard, O. et al. 2009, A\& A, 502, 771

Dolag, K., Murante, G., \& Borgani, S. 2010, MNRAS 405, 1544

D'Souza, R., Kauffman, G., Wang, J., \& Vegetti, S. 2014, MNRAS 443, 1433

Feldmeier, J. J., Ciardullo, R., \& Jacoby, G. H. 1998, ApJ 503, 109

Feldmeier, J. J., Ciardullo, R., Jacoby, G. H., \& Durrel, P. 2003, ApJS 145, 65

Feldmeier, J. J., Ciardullo, R., Jacoby, G. H. et al. 2004, ApJ, 615, 196

Gerhard, O. E., Arnaboldi, M., Freeman, K. C., Okamura, S. et al. 2007, A\&SA, 498, 815

Gerhard, O. E., Arnaboldi, M., Freeman, K. C., Kashikawa, N. et al. 2005, ApJ, 621, L93

Jacoby, G. H. \& De Marco, O. 2002, AJ, 123, 269

Kormendy, J., Fisher, D. B., Cornell, M. E., \& Bender, R. 2009, ApJS, 182, 216

Longobardi, A., Arnaboldi, M., Gerhard, O., \& Hanuschik, R. 2015, A\& A 579, 135

Longobardi, A., Arnaboldi, M., Gerhard, O., Coccato, L. et al. 2013, A\&A 558, 42

Mihos, J. C. 2015 IAU Symp. 317, 2015, arXiv.1510.01929

Murante, G., Arnaboldi, M., Gerhard, O., Borgani, S. et al. 2004, ApJL 607, 83

Murante, G., Giovalli, M., Gerhard, O., Arnaboldi, M., et al. 2007, MNRAS, 377, 2

Puchwein, E., Springel, V., Sijacki, D., \& Dolag, K. 2010, MNRAS 406, 936

Reid, W. A. \& Parker, Q. A. 2010, MNRAS, 405, 1349

Rudick, C. S., Mihos, J. C., \& McBride, C. 2006, ApJ, 648, 936

Rudick, C. S., Mihos, J. C., Frey, L. H., McBride, C. K. 2009, ApJ, 699, 1518

Thuan, T. X. \& Kormendy, J. 1977, PASP, 89, 466

Ventimiglia, G., Arnaboldi, M., Gerhard, O. E. 2011, A\& A, 528, 24

Zibetti, S., White, S. D. M., Schneider, D. P., \& Brinkmann, J. 2005, MNRAS 358, 949

White, S. D. M.\& Rees, M. J. 1978, MNRAS, 183, 341 